\title{
ÉTUDE SUR LES CAPACITÉS DE FRANCHISSEMENT DES CABOTS BOUCHE-RONDES (SICYOPTERUS LAGOCEPHALUS, PALLAS, 1770) EN VUE DE LA CONCEPTION DE DISPOSITIFS ADAPTÉS AUX PRISES D'EAU DU TRANSFERT SALAZIE (ÎLE DE LA REUNION).
}

\author{
B. VOEGTLÉ (1), M. LARINIER (2), P. BOSC (3)
}

(1) SIEE Sud Ouest, Parc technologique du canal, 2 rue Giotto, 31520 RAMONVILLE SAINT AGNE, France. E-mail : bruno.voegtle@siee.fr

(2) CSP-CEMAGREF-GHAAPPE, Institut de Mécanique des Fluides, Avenue du Professeur Camille Soula, 31400 TOULOUSE, France. E-mail : larinier@imft.fr

(3) ARDA, Route forestière de l'Etang du Gol, 97427 ÉTANG SALÉ (île de la Réunion), France. E-mail : arda@guetali.fr

\section{RÉSUMÉ}

L'espèce de gobiidae amphidrome "cabot bouche-ronde " (Sicyopterus lagocephalus, Pallas, 1770) effectue au stade postlarvaire des migrations de masse aux embouchures des rivières de l'île de la Réunion. Elle fait l'objet lors de cette migration d'une pêche artisanale qui demeure encore aujourd'hui tout à fait significative sur le plan socio-économique. La préservation de l'espèce et de la pêcherie nécessite que demeure garanti l'accès à ses zones de croissance et de reproduction.

Un projet de captage des eaux consistant à transférer à partir des cirques de Mafate et Salazie (région la plus arrosée de l'île) les quantités d'eau nécessaires aux besoins de la région Ouest (région la plus sèche) est en cours. Ce captage devait être initialement effectué par des prises d'eau qui auraient constitué des obstacles infranchissables à la migration du cabot bouche-ronde.

Afin de concevoir sur ces prises d'eau des dispositifs de franchissement adaptés à cette espèce, une étude sur pilote expérimental a été effectuée pour mieux appréhender ses capacités de franchissement. L'installation consiste en 5 rampes à pentes variables installées en parallèle susceptibles d'être alimentées par des débits variables.

$\mathrm{Au}$ cours de cette étude, 7 revêtements (béton lisse, béton rugueux, béton cyclopéen, deux substrats de béton lisse comportant des bosses, PVC et tôle), 6 charges (variant de $1 \mathrm{~mm}$ à $24 \mathrm{~mm}$ ) et 3 pentes $\left(50^{\circ}, 70^{\circ}\right.$ et $\left.90^{\circ}\right)$ ont été testés sur des lots comportant des individus de tailles significativement différentes.

Les capacités de progression sont fortement liées à la taille des individus. Le débit peut bloquer, dès qu'il devient trop important, toute montaison. Bien que les poissons 
puissent franchir un mur vertical, le pourcentage de passage et le taux de réussite est beaucoup plus faible que pour les autres pentes testées. Une pente à $50^{\circ}$, et une charge de l'ordre de 1-2 mm sont les deux conditions ayant permis sur le pilote d'obtenir les meilleurs résultats. Le revêtement de la rampe s'est révélé être un facteur secondaire, le béton lisse constituant cependant le meilleur support.

Les résultats de cette étude ont permis de proposer des critères de dimensionnement pour des dispositifs de franchissement adaptés aux sites et à l'espèce. La passe consiste en une rampe en béton lisse installée à une pente de $50^{\circ}$ alimentée par déversement. Une alimentation non uniforme sur la largeur de la rampe permet d'obtenir, quel que soit le niveau amont, une zone favorable à la remontée du poisson.

Mots-clés : montaison, prise d'eau, Cabot bouche-ronde, Sicyopterus lagocephalus, dispositif expérimental, capacités de reptation.

\section{EXPERIMENTAL STUDY OF THE CLIMBING CAPABILITIES OF THE GOBY SICYOPTERUS LAGOCEPHALUS (PALLAS, 1770) FOR THE DESIGN OF UPSTREAM FACILITIES AT THE SALAZIE DIVERSION WATER INTAKES (REUNION ISLAND).}

\section{ABSTRACT}

The postlarvae of amphidromous goby Sicyopterus lagocephalus (Pallas, 1770) are known to start their migration at the mouths of rivers in the Reunion Island. This mass migration provides a major artisanal fishing resource of noteworthy socio-economic importance. A necessary condition for protecting the resource is the preservation of the fishes' free access to the breeding and spawning areas.

A water diversion project has been undertaken for transferring water from the wettest part of the Island (Salazie and Mafate cirques) to the driest part (the West region). Fish passage facilities must be built at these water intakes which will otherwise impede the upstream movement of migratory species, namely Sicyopterus lagocephalus.

An experimental study has been performed to find out more about the climbing capabilities of this species. The test bench consists of 5 ramps which can be fed with variable discharges. Seven substrate types (smooth and rough concrete, smooth concrete with two sizes of bumps, masonry, PVC, metal), 6 upstream heads and three slopes have been tested with batches of different sized fish.

The climbing capabilities are strongly related to the length of the fish. If an upstream head feeding the ramp is to great this may impede fish passage. The rate of passage is significantly lower for the vertical ramp than for the two gentler slopes tested. The $50^{\circ}$ slope and a head of $1-2 \mathrm{~mm}$ are the two conditions giving the best results. The substrate covering the ramp is of little importance, although smooth concrete seems to be the best substrate.

Analysis of the results led to design criteria for fish facilities which are adapted to the species and water intake constraints.

Key-words : upstream migration, water intake, Sicyopterus lagocephalus, experimental flume, climbing capabilities. 


\section{INTRODUCTION}

La pêche du bichique, juvénile du cabot bouche-ronde (Sicyopterus lagocephalus, Pallas, 1770) est une activité traditionnelle sur l'île de la Réunion qui demeure encore aujourd'hui tout à fait significative sur le plan socio-économique. Les cabots bouche-rondes remontent les cours d'eau pour y effectuer la quasi-totalité de leur croissance et s'y reproduire. La préservation de l'espèce nécessite que l'accès à leurs zones de croissance et de reproduction soit garanti. On a constaté depuis une dizaine d'années une baisse inquiétante de la ressource, qui peut être attribuée à l'augmentation de la pression de pêche, à la répétition des sécheresses, ainsi qu'au développement des ouvrages de captage en rivière, qui constituent le plus souvent des obstacles infranchissables à la colonisation des zones amont des rivières pour les diverses espèces de poissons et de crustacés (DELACROIX, 1992).

Un projet majeur de transfert des eaux a été initié il y a une quinzaine d'années par le Département de la Réunion. II consiste à capter et à transférer à partir des régions les plus arrosées de l'île (cirques de Mafate et de Salazie), les quantités d'eau nécessaires aux besoins de la région Ouest, beaucoup plus sèche. Ce captage des eaux sera à terme effectué par quatre ouvrages de conception très voisine, constitués par un seuil assurant la coupure de la rivière dans lequel est incorporée une prise d'eau " en-dessous ». Ces seuils, d'une dizaine de mètres de hauteur, constituent un obstacle total pour la migration des poissons, et plus particulièrement des cabots bouche-rondes.

L'étude menée au sein du Centre des Eaux Douces de l'ARDA (Association Réunionnaise pour le Développement de l'Aquaculture) et décrite dans la suite était destinée à acquérir les informations minimales sur les capacités de franchissement des cabots bouche-rondes permettant d'établir les critères de dimensionnement des futurs dispositifs de franchissement à aménager sur les ouvrages de prises d'eau. Dans le cadre de cette étude, un pilote expérimental a été construit afin d'étudier plus en détail les comportements et les capacités de franchissement de cette espèce et de préciser les conditions hydrauliques favorables à leur progression sur un obstacle.

\section{ESPÈCES ET PARTICULARITÉS PHYSIQUES DU CABOT BOUCHE-RONDE}

« Cabot bouche-ronde » est le nom local donné à l'adulte (longueur supérieure à $35 \mathrm{~mm}$ ) du Sicyopterus lagocephalus (Pallas, 1770), espèce indigène de la famille des Gobiidae présente dans les rivières de l'île (DELACROIX, 1992 ; BOSC et al., 1996). Cette espèce, dont le juvénile est appelé " bichique ", est une espèce amphidrome. Les adultes sont très rhéophiles et vivent en rivière dans les zones rapides sur les fonds de cailloux et de galets (Photo 1). Ils se reproduisent en rivière du mois de janvier au mois de juin. Le développement embryonnaire se fait en eau douce et les larves doivent passer en eau de mer dans les jours qui suivent l'éclosion des œufs (VALADE et al., 2001). Les larves se développent et deviennent des postlarves pélagiques en mer. A une taille d'environ $20 \mathrm{~mm}$, elles se regroupent aux embouchures pour effectuer leur migration anadrome au cours de laquelle elles se métamorphosent en juvéniles benthiques. Les poissons colonisent les cours d'eau jusqu'à une altitude supérieure à $600 \mathrm{~m}$, voire $800 \mathrm{~m}$ (KEITH et al., 1999).

Cette espèce se caractérise par une ventouse subdiscoïdale formée par la coalescence des nageoires pelviennes. Cet organe lui confère des capacités exceptionnelles de franchissement des obstacles naturels, comme le démontre la distribution longitudinale de l'espèce sur les différents cours d'eau de l'île de la Réunion (BOSC et al., 1998 ; RICOU et al., 1999). 
II n'existe néanmoins que très peu de données objectives sur les conditions de franchissement in situ des obstacles par les cabots bouche-rondes, sachant que ces informations sont très difficiles à acquérir sans recours à des moyens logistiques et humains très lourds compte tenu de l'extrême variabilité des facteurs susceptibles d'influencer le franchissement d'un obstacle en milieu naturel : convergence d'un flux migratoire et de conditions hydrologiques favorables au franchissement de l'obstacle, état physiologique du poisson, hétérogénéité de la géométrie des obstacles et difficulté d'appréhension des conditions hydrauliques.

Pour définir les capacités de franchissement des cabots bouche-rondes, il a semblé préférable de privilégier une expérimentation en laboratoire permettant de contrôler certains facteurs susceptibles de se révéler déterminants, comme les conditions hydrauliques (débit, vitesse d'écoulement, pente...), la nature du substrat, certains paramètres environnementaux (luminosité...) et enfin le stade biologique (taille des individus).

\section{MATÉRIEL ET MÉTHODES}

\section{Dispositif expérimental}

Le pilote est constitué de 5 rampes parallèles de $15 \mathrm{~cm}$ de largeur et de $4 \mathrm{~m}$ de longueur, à inclinaison variable. Leur pied est situé dans un bassin circulaire d'une profondeur d'une cinquantaine de $\mathrm{cm}$. Situé en tête des rampes, un bac muni d'un déversoir permet une alimentation des cinq rampes par un débit identique (Photos 2 et 3 ).

Les poissons lâchés dans le bassin aval, au pied des rampes, ont la possibilité de choisir eux-mêmes leur support lorsque toutes les rampes sont alimentées. Une fois arrivés en haut des rampes, ils sont piégés dans un vivier de stockage propre à chaque rampe (Photo 4).

Une pompe permet de refouler l'eau du bac inférieur jusqu'au bac supérieur à un débit constant voisin de $5 \mathrm{l} / \mathrm{s}$. Un tuyau PVC muni d'une vanne et reliant le bac supérieur au bac inférieur permet le réglage du débit passant par les rampes.

Une alimentation permanente en eau provenant de la rivière St Etienne, non traitée, permet de conserver la température et la qualité de l'eau constantes au cours des essais.

Pour permettre une alimentation constante et régulière des 5 rampes, la sortie du tuyau d'alimentation en eau du bac supérieur est équipée d'une crépine et des flotteurs constitués de plaques en bois permettent d'amortir les ondes de surface (Photo 4).

\section{Matériel vivant}

Les cabots bouche-rondes sont des poissons assez faciles à transporter. Par contre, la difficulté à les alimenter ne facilitant pas leur maintien en captivité, il s'est avéré indispensable de renouveler à intervalles réguliers par pêches électriques les lots de poissons utilisés pour les tests afin de limiter le biais de comportement induit par leur stabulation en bassin et la diminution progressive de leur activité migratoire.

Les essais ont été réalisés avec 20 lots différents de poissons sauvages. Chaque lot de poissons a subi 3,7 essais en moyenne. 


\section{Protocole expérimental}

Afin de tester l'influence du support, 5 rampes en matériaux différents ont été construites. Le choix s'est porté initialement vers les supports suivants :

- PVC : support témoin par sa surface lisse et permettant au cabot bouche-ronde de très bien se ventouser.

- tôle : il a semblé intéressant de tester un support en tôle car ne sachant pas au départ la position de la passe par rapport à la prise d'eau, une passe en acier aurait pu être envisagée pour résister au transport solide*.

- béton lisse et béton rugueux : le béton est le matériau de prédilection utilisé en génie civil. La rugosité de la surface joue sur les caractéristiques hydrauliques de l'écoulement.

- béton " cyclopéen » : ce support comporte des galets de basalte partiellement noyés dans un béton rugueux. Les galets ressortant à la surface sont susceptibles de créer des zones d'abris hydrauliques pour les poissons.

Les premiers essais ont été effectués à une pente de $70^{\circ}$. Suite aux résultats obtenus à cette pente, les deux supports en tôle et en PVC ont été supprimés et remplacés par deux nouveaux supports en béton lisse comportant des renflements (bosses) de tailles et dimensions différentes : un " béton grosses bosses " (diamètre moyen environ $10 \mathrm{~cm}$, hauteur $3 \mathrm{~cm}$ ) et un " béton petites bosses " (diamètre moyen environ $5 \mathrm{~cm}$, hauteur $2 \mathrm{~cm}$ ). Les 5 rampes ont été installées ensuite à $50^{\circ}$ et $90^{\circ}$.

Pour chacune des trois pentes, les rampes ont été alimentées avec des charges variant entre $1 \mathrm{~mm}$ et $24 \mathrm{~mm}$. Les débits ont été estimés soit par jaugeage pour les plus faibles, soit en utilisant une formule de type déversoir à partir de la charge hydraulique lue dans le bac supérieur. Au total, 6 charges correspondant à 6 débits ont été testées : $1 \mathrm{~mm}$ $(0,056 \mathrm{l} / \mathrm{s} / \mathrm{m}), 2 \mathrm{~mm}(0,17 \mathrm{l} / \mathrm{s} / \mathrm{m}), 4 \mathrm{~mm}(0,47 \mathrm{l} / \mathrm{s} / \mathrm{m}), 8 \mathrm{~mm}(1,27 \mathrm{l} / \mathrm{s} / \mathrm{m}), 15 \mathrm{~mm}(3,33 \mathrm{l} / \mathrm{s} / \mathrm{m})$ et $24 \mathrm{~mm}(6,66 \mathrm{l} / \mathrm{s} / \mathrm{m})$.

Les essais ont été réalisés de jour et de nuit, avec ou sans éclairage.

La durée d'un essai a été fixée dans un premier temps à 24 heures, pour permettre d'intégrer le rythme nycthéméral éventuel des poissons. Suite aux 18 premiers essais, la durée des essais a pu être réduite à 10 heures.

Les essais ont généralement été doublés. Ils ont été filmés à l'aide d'une caméra placée face aux rampes. Les enregistrements vidéo permettent non seulement d'observer en détail le comportement de progression des cabots bouche-rondes mais aussi de noter le nombre de tentatives de montée par support. On peut comparer ce nombre au nombre d'individus piégés dans le bac supérieur, ce qui donne un indice sur le taux de réussite du franchissement. Ce nombre de tentatives observées rapporté au nombre de poissons lâchés dans le bac inférieur donnera également un « indice d'activité » pour chaque lot.

71 essais ont été réalisés entre le 9 avril et 10 juillet 1998 dont 21 à la pente $70^{\circ}$, 32 à la pente $50^{\circ}$ et 18 à la pente $90^{\circ}$.

\footnotetext{
* Les parties déversantes des seuils de prise d'eau sont en effet revêtues d'un blindage d'acier de $2 \mathrm{~cm}$ pour résister à l'érosion induite par le transport solide en crue.
} 
Pour chaque essai, on a relevé le nombre et la taille des individus lâchés dans le bassin aval ; il en a été fait de même pour les individus récupérés dans les pièges amont. Les enregistrements vidéo ont permis de comptabiliser l'évolution des passages en pied de rampes en fonction du temps. A partir des données brutes obtenues, on a cherché à mettre en évidence l'influence du sexe, du support, du débit, de la pente, de la taille des poissons et de la lumière, sur le pourcentage de poissons montés (à partir des piégeages à l'amont des rampes) et sur le pourcentage de réussites par rapport aux tentatives (à partir des observations vidéo).

\section{RÉSULTATS}

\section{Mode de progression du cabot bouche-ronde}

La progression est lente et se fait $\mathrm{cm}$ par $\mathrm{cm}$ en plusieurs phases, le cabot utilisant successivement sa bouche et son disque pelvien comme ventouse pour se fixer sur le support en s'aidant des nageoires pectorales.

Sur pilote expérimental comme en milieu naturel, on peut observer que les cabots bouche-rondes ont un comportement de groupe. Leur montaison en groupe facilite le franchissement des obstacles. Les individus de tête créent un sillage dans l'écoulement dont bénéficie le reste du groupe (Photo 5).

De plus, le comportement des poissons est grégaire : il suffit souvent qu'un individu du groupe se laisse décrocher pour que ceux situés en aval décrochent à leur tour, alors que les conditions hydrauliques dans lesquelles ils se trouvent n'ont pas sensiblement évolué.

\section{Evolution des tentatives de montées au cours du temps}

Les poissons montent essentiellement lors des premières heures de l'essai. Lorsque l'on examine l'évolution du nombre de tentatives en fonction du temps sur les 18 premiers essais, on voit que plus de $90 \%$ des tentatives ont lieu pendant les 7 premières heures. Sur la totalité des essais effectués, $45 \%$ des tentatives se produisent durant la première heure de l'essai.

Suite à ces observations, la durée des essais a été réduite de $24 \mathrm{~h}$ à $10 \mathrm{~h}$ pour limiter ainsi au maximum le stress des poissons.

\section{Influence du sexe}

Lors des 12 premiers essais, l'influence éventuelle du sexe sur le comportement de montaison a été examinée. Même si le pourcentage de femelles montées semble plus important (47,9 \% pour les femelles et $43,3 \%$ pour les mâles), la différence n'est pas significative $(F=0,59, P=0,306)$. Lors des essais ultérieurs, le sexe des individus n'a plus été relevé, ce qui a permis de réduire la manipulation des poissons, et par conséquent les risques de stress et de blessure.

\section{Influence du support}

L'état de surface peut avoir une influence sur la facilité que possèdent les poissons à se ventouser. Des analyses de variance ont été effectuées afin de déterminer l'efficacité de chaque support. 


\section{Pente $70^{\circ}$}

L'influence de cinq supports (béton lisse, tôle, béton cyclopéen, PVC, béton rugueux) sur le pourcentage de montée a été testée, lorsque toutes les rampes sont alimentées en même temps et pour plusieurs débits d'alimentation.

II existe une influence significative du support $(F=30,4, P=0,00)$. Le béton lisse est choisi préférentiellement, avec $58,7 \%$ de poissons montés. Le béton rugueux vient en deuxième position avec $25 \%$ de poissons montés. Les deux rampes au support très lisse (tôle et PVC) sont très peu empruntées par les poissons (moins de $5 \%$ des individus). Les cabots qui choisissent ces substrats très lisses semblent éprouver beaucoup plus de difficultés à monter et à lutter contre le courant que sur les autres substrats. Le béton cyclopéen est également très peu choisi ( $8 \%$ des individus), les poissons ne semblant cependant pas éprouver de difficulté particulière à monter. Ce résultat est probablement induit par un effet de bord. En effet, les cabots semblent choisir préférentiellement les supports situés aux deux extrémités, à savoir les bétons lisses et rugueux.

C'est pourquoi, dans un deuxième temps, il a été décidé de tester les 3 rampes en béton une par une, en ne laissant pas le choix du support aux poissons et en supprimant tout effet de bord éventuel. Tous les supports ont été alimentés par un débit identique correspondant à une charge de $4 \mathrm{~mm}$.

Dans ces conditions d'alimentation, le béton lisse et le béton cyclopéen deviennent les support les plus efficaces, avec respectivement $40,3 \%$ et $36,7 \%$ de poissons montés. Ces supports s'avèrent nettement plus efficaces que le béton rugueux $(7,8 \%)$.

Les aspérités du béton rugueux empêchent les poissons de ventouser parfaitement. Ce phénomène est particulièrement visible pour les gros individus. Le béton cyclopéen se révèle relativement bon ; les rugosités du béton situées entre les galets étant cependant susceptibles de réduire l'efficacité du ventousage des poissons. C'est pourquoi, dans la suite des essais, les supports en tôle et en PVC ont été remplacés par deux nouveaux supports : le " béton grosses bosses " et le " béton petites bosses ». Ces deux supports sont des compromis entre le béton lisse et le béton cyclopéen, puisqu'ils présentent un revêtement lisse pour permettre un bon ventousage des poissons et des bosses plus ou moins grosses pour créer une diversité des conditions hydrauliques susceptible de favoriser la progression du poisson.

\section{Autres pentes : $50^{\circ}$ et $90^{\circ}$}

Lorsque toutes les rampes sont alimentées en même temps, l'analyse de variance montre qu'à $50^{\circ}$, le support influence le pourcentage de montées $(F=42,9, P=0,00)$. Le béton lisse est choisi préférentiellement, avec $60 \%$ de poissons montés. Le béton rugueux vient en deuxième position avec $26,7 \%$ de poissons montés. Les 3 autres bétons sont très peu empruntés par les cabots (moins de $6 \%$ de poissons montés). Comme pour la pente de $70^{\circ}$, ce résultat est manifestement induit par un effet de bord.

Les différents supports ont été par la suite testés un par un afin de supprimer l'effet de bord. Pour les deux pentes de $50^{\circ}$ et $90^{\circ}$, le béton lisse reste encore une fois le support le plus efficace ( $44 \%$ de poissons montés), le béton rugueux devenant le moins bon support avec $24 \%$ de poissons montés.

En conclusion, quelles que soient la pente et les conditions d'alimentation, le support semble avoir une influence certaine, mais moins déterminante que présumée. Les poissons préfèrent les surfaces ni trop lisses (comme le PVC, qui facilite la fixation du poisson mais génère des vitesses d'écoulement importantes), ni trop rugueuses (comme le béton rugueux, qui rend la fixation du poisson difficile). Le béton lisse reste toujours le support le plus emprunté par les poissons, ce résultat demeurant valable pour toutes les classes de taille. 


\section{Influence du débit}

Une analyse de variance a été effectuée afin de déterminer l'influence du débit sur le pourcentage de montée en prenant les résultats obtenus quelle que soit la pente et lorsque toutes les rampes sont alimentées en même temps.

Les charges testées ont été regroupées en trois classes : une classe de faible charge qui regroupe les valeurs des charges de $1 \mathrm{~mm}$ et $2 \mathrm{~mm}$, une classe de charge moyenne regroupant les valeurs de $4 \mathrm{~mm}$ et $8 \mathrm{~mm}$, et une classe de forte charge qui regroupe les valeurs des charges de $15 \mathrm{~mm}$ et $24 \mathrm{~mm}$.

Pour la classe inférieure de charge, le pourcentage de poissons montés est de $56 \%$. Il passe à $37,1 \%$ pour la classe de charge moyenne et à $27,3 \%$ pour la classe de forte charge. Le pourcentage de montée diminue nettement lorsque la charge augmente. Si l'effet de la charge sur le comportement du poisson n'est pas statistiquement significatif $(F=2,23 ; P=0,13)$, il est visuellement très marqué.

La montaison est meilleure lorsque le cabot bouche-ronde bénéficie d'un support juste humidifié. II suffit d'une charge de $15 \mathrm{~mm}$, c'est à dire d'un débit de $3,33 \mathrm{l} / \mathrm{s} / \mathrm{m}$, pour réduire de moitié le nombre de poissons montés.

\section{Influence de la pente}

Une analyse a été effectuée afin de déterminer l'influence de la pente sur le pourcentage de montée en prenant les résultats obtenus quel que soit le débit, lorsque toutes les rampes sont alimentées en même temps.

L'analyse montre que la pente influence le pourcentage de montées des poissons $(F=3,857, P=0,04)$. On peut constater que le pourcentage de montées est d'autant plus faible que la pente est forte. En moyenne, $58 \%$ des poissons sont montés à $50^{\circ}, 45 \%$ sont montés à $70^{\circ}$ et seulement $20 \%$ sont montés à $90^{\circ}$.

La pente est, avec le débit, le facteur qui semble le plus influencer la réussite de la montaison des poissons.

\section{Influence de la taille}

Des analyses de variances ont été effectuées à partir des résultats obtenus pour les pentes extrêmes $\left(50^{\circ}\right.$ et $\left.90^{\circ}\right)$ pour mettre en évidence l'influence de la taille des poissons sur le pourcentage de montées.

Le pourcentage de montée diminue rapidement lorsque la taille augmente. A la pente $50^{\circ}$, les résultats obtenus sont les suivants: $51,8 \%$ des poissons de taille 40-60 mm, 37,8 \% de la classe $60-80 \mathrm{~mm}$ et seulement $23 \%$ de la classe sup. $80 \mathrm{~mm}$, sont montés. Les pourcentages de montées à la pente $90^{\circ}$ sont les suivants : $23,9 \%$ pour la classe de taille $40-60 \mathrm{~mm}, 14,5 \%$ pour la classe de taille $60-80 \mathrm{~mm}$ et seulement $1 \%$ pour la classe de taille sup. $80 \mathrm{~mm}$. L'influence du facteur taille est donc très nette quelle que soit la pente considérée.

\section{Rythme nycthéméral et influence de l'éclairage}

Des essais ont été réalisés de jour et de nuit, avec ou sans éclairage. Les résultats semblent indiquer que les poissons montent aussi bien de nuit sans éclairage $(26,4 \%$ d'individus montés) que de jour (31\%) ou de nuit avec éclairage (31\%). 


\section{Interactions entre les différents facteurs}

\section{Interaction entre la pente et la charge}

Une analyse de variance à deux facteurs a été réalisée afin de déterminer l'interaction de la charge amont et de la pente sur le pourcentage de montées. Cette analyse a été effectuée avec les trois classes de charge : faible, moyenne et forte

Pour la pente $50^{\circ}$, la charge influence peu le pourcentage de montées qui reste relativement élevé quel que soit le débit (entre $53,1 \%$ et $68,8 \%$ ). Par contre, pour la pente $70^{\circ}$, une forte charge entraîne une baisse importante du pourcentage de montées. A la pente de $90^{\circ}$, seule la charge la plus faible permet d'obtenir des pourcentages de montées satisfaisants, les débits moyen et fort empêchant les poissons de monter. Quelle que soit la pente, une charge faible admet toujours un même pourcentage de montées d'environ $55 \%$. Une réduction de la pente augmente la plage des débits permettant le franchissement des cabots bouche-rondes. Cette interaction est cependant peu significative $(F=3,021, P=0,057)$.

Interaction entre les classes de taille, les débits et les pentes

II n'existe aucune interaction significative sur les pourcentages de montées entre les classes de taille et les débits, ni entre les classes de taille et les pentes ( $F=1,74$, $P=0,14),(F=0,413, P=0,80)$.

\section{Remarques sur les capacités de franchissement du Cotylopus acutipinnis}

Si Sicyopterus lagocephalus domine largement le peuplement de Gobiidae migrateur des rivières de La Réunion en terme d'effectif, il existe toutefois une deuxième espèce, Cotylopus acutipinnis (Guichenot, 1863), appelé aussi cabot bouche-ronde ou cabot de cascade, et souvent confondu avec Sicyopterus lagocephalus. La présence de trois encoches sur la lèvre supérieure de Sicyopterus lagocephalus est un signe distinctif. Cotylopus acutipinnis a par ailleurs un corps plus fin et le mâle adulte est beaucoup moins coloré.

Les deux espèces présentent les mêmes exigences d'habitat et leur cycle biologique est vraisemblablement très proche (DELACROIX,1992 ; BOSC et al., 1996 ; KEITH et al., 1999).

L'étude a permis d'observer les capacités de franchissement de cette espèce. II en ressort principalement que le Cotylopus acutipinnis utilise peu sa bouche en complément de sa ventouse pelvienne pour se fixer et progresser le long d'une paroi, mais compense par des mouvements de reptation de son corps.

II semblerait cependant que le Sicyopterus lagocephalus monte relativement mieux que le Cotylopus acutipinnis (37,5\% contre $28,8 \%$, tous les essais confondus), ce qui confirme les observations effectuées en milieu naturel et lors des pêches amont et aval d'obstacles (BOSC et al., 1998). La différence n'est cependant pas significative ( $F=2,67$, $P=0,106)$.

\section{DISCUSSION ET CONCLUSION}

Même si l'on connaissait les prédispositions particulières au franchissement d'obstacles que possèdent les cabots bouche-rondes, les expérimentations menées sur pilote ont permis de caractériser les modes de progression et les capacités de 
franchissement de ces poissons et de préciser les conditions hydrauliques permettant leur montaison.

Les résultats obtenus sont toutefois à analyser avec précaution sachant que l'on ne peut pas écarter l'existence de biais liés aux conditions expérimentales (effet de confinement des lots testés...).

Parmi les principaux résultats, on retiendra notamment :

- le débit et la pente sont des facteurs particulièrement importants, pouvant lorsqu'ils sont trop élevés, interdire totalement la montaison des poissons,

- la plage de débit pour laquelle un support est franchissable est d'autant plus faible que la pente est importante,

- le revêtement de surface, s'il influence les capacités de franchissement, est moins discriminant que présumé ; le béton lisse semble toutefois être le plus efficace de tous les supports testés,

- la taille des individus est un paramètre important à considérer car il influe sur les capacités de franchissement. Les adultes d'une taille supérieure à $80 \mathrm{~mm}$ éprouvent en effet de grandes difficultés à franchir les obstacles. Les poissons appartenant à la classe de taille 40-60 $\mathrm{mm}$ apparaissent détenir les meilleures aptitudes au franchissement.

L'exploitation des résultats précédents, complétée par les observations recueillies en milieu naturel (BARIL, 1993), ainsi que les contraintes constructives ont permis de définir les caractéristiques des dispositifs de franchissement des prises d'eau situées sur les rivières du Mât et des Fleurs Jaunes (Cirque de Salazie).

Les crues cycloniques et le transport solide très intense des rivières réunionnaises font que l'installation d'une passe à poissons classique n'est pas envisageable. Seul un dispositif de franchissement d'emprise très limitée, ne demandant aucun entretien particulier et utilisant les capacités de franchissement exceptionnelles des cabots boucherondes pouvait être envisagé.

Le dispositif de franchissement a été prévu à l'intérieur de l'ouvrage de prise pour limiter son exposition aux crues. La passe consiste en un plan incliné en béton lisse, de pente $50^{\circ}$. L'alimentation se fait par déversement gravitaire, la cote de déversement n'est pas horizontale. Cette alimentation non-uniforme de la rampe sur plus de $4 \mathrm{~m}$ de largeur permet de créer en permanence, pour les conditions de niveaux d'eau à l'amont de l'ouvrage de prise les plus fréquentes, une zone très faiblement alimentée favorable à la remontée du poisson (BRL, 1999).

Un canalet, muni d'une grille de protection à son entrée destinée à retenir les éléments les plus grossiers, alimente la passe. Une vanne ainsi qu'une légère pente du radier du canalet permettront l'évacuation par chasse hydraulique des éléments les plus fins déposés dans celui-ci.

Si l'on peut raisonnablement penser que la franchissabilité vers l'amont des futurs ouvrages de prises d'eau lors de la migration anadrome des cabots bouche-rondes ne devrait plus poser de difficultés, le problème de la dévalaison des larves reste entier.

Au vu de la faible mobilité de ces larves, la proportion des larves entraînées dans les prises d'eau devrait être fonction du rapport du débit prélevé sur le débit naturel (VALADE, 2001a). Compte tenu du mode de gestion prévu et de l'importance des débits prélevés, il est à prévoir que, sans dispositif et/ou mesures de gestion particulières 
adoptées lors des périodes de dévalaison de ces larves, l'impact des prises d'eau risque d'être majeur et réduire à néant les efforts consentis pour résoudre la migration anadrome des adultes.

C'est pourquoi une étude a été initiée pour acquérir une meilleure connaissance du cycle de reproduction du cabot bouche-ronde et des facteurs environnementaux (débit, température, cycle lunaire, disponibilité trophiques...) susceptibles d'influer sur le cycle sexuel (maturation des géniteurs, ponte, incubation des œufs...), ainsi que des conditions de dévalaison des larves au niveau éco-éthologique et biologique. D'autre part une étude sur dispositif expérimental modélisant les conditions d'écoulement dans les décanteurs de prises d'eau est en cours. Elle permettra notamment de tester le comportement des larves dans l'écoulement (portance, sédimentation...) et d'évaluer la faisabilité d'un dispositif de récupération (VALADE, 2001b).

\section{REMERCIEMENTS}

Nous tenons à remercier l'ensemble du personnel du Centre des Eaux Douces de l'ARDA pour leur aide technique apportée lors de la construction du dispositif expérimental et au cours des campagnes de pêches électriques. Le Conseil Général, la DIREN de la Réunion et EDF ont assuré conjointement la maîtrise d'ouvrage de l'étude.

\section{BIBLIOGRAPHIE}

BARIL D., 1993. Prises d'eau de Mafate - Rivière des Galets et bras de Sainte Suzanne Rapport d'expertise. Conseil Supérieur de la Pêche, Délégation Régionale Languedoc Roussillon, $8 \mathrm{p}$.

BOSC P., DELACROIX P., FERRAND P., MABILLE A., 1996. Inventaire Piscicole des cours d'eau pérennes de l'île de la Réunion. Rapport ARDA DIREN, 124 p.

BOSC P., LARINIER M., VOEGTLÉ B., 1998. Etude sur les capacités de franchissement des cabots bouche-rondes (Sicyopterus lagocephalus) en vue de la conception de dispositifs adaptés aux prises d'eau du transfert Salazie (île de la Réunion), $44 \mathrm{p}$.

BRL, 1999. Irrigation du littoral Ouest - Dossier de demande d'autorisation au titre de la loi sur l'eau, documents d'incidences, $97 \mathrm{p}$.

DELACROIX P., 1992. Irrigation du littoral Ouest - Transfert Salazie. Etude complémentaire de la biologie, de la répartition et des niveaux de capture des population de bichiques de la rivière du Mât, $66 \mathrm{p}$.

KEITH P., VIGNEUX E., BOSC P., 1999. Atlas des poissons et des crustacés d'eau douce de la Réunion. Patrimoines naturels, (M.N.H.N./S.P.N.), 39, 136 p.

RICOU J.F., BOSC P., CADENE R., 1999. Mise en place d'un Réseau Piscicole à La Réunion. Adaptation méthodologique d'un protocole d'échantillonnage de l'ichtyofaune. Rapport final d'étude, 100 p.

VALADE P., 2001a. Etude des capacités de franchissement des ouvrages de captage d'eau du transfert Est-Ouest par les larves et les œufs des cabots bouche-rondes à la Réunion. Rapport ARDA, Conseil Général, $31 \mathrm{p}$.

VALADE, 2001b. Etude de la reproduction et des premiers stades larvaires du cabot bouche-ronde à l'île de la Réunion en vue de la conception d'aménagements et de mesures de gestion favorisant le franchissement des ouvrages de captage d'eau par les larves lors de la migration d'avalaison. Diplôme de Recherche Technologique, Université de Montpellier II, $135 \mathrm{p}$.

VALADE P., RICOU J.F., GRONDIN PH., BOSC P., 2001. Etude de la biologie de la reproduction et du cycle larvaire du bouche ronde, Sicyopterus lagocephalus (Pallas, 1770) et Cotylopus acutipinnis (Guichenot, 1863). Description des premiers stades larvaires, étude du comportement et de la résistance des larves en eau douce et en eau de mer. Rapport ARDA, 54 p. 


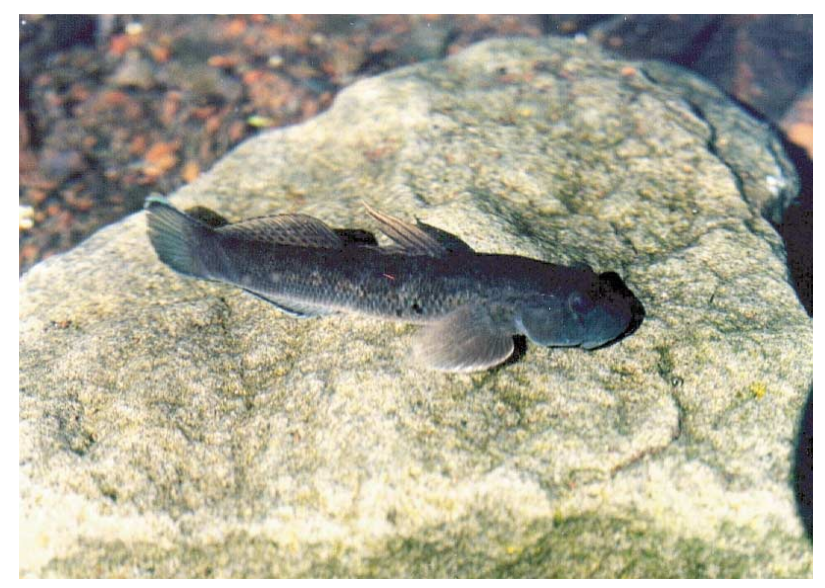

Photo 1 : Sicyopterus lagocephalus (femelle). Photo 1 : Sicyopterus lagocephalus (female).

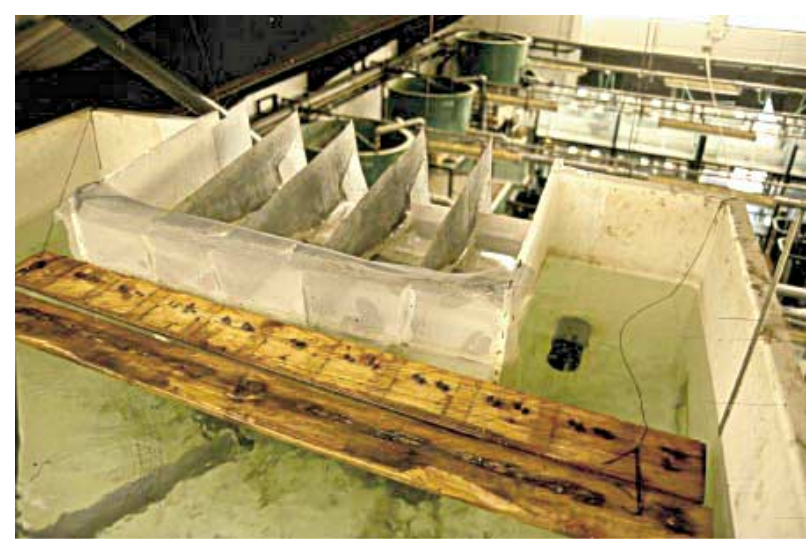

Photo 4 : vue du bassin amont montrant les nasses de piégeage sur chaque rampe.

Photo 4 : view from the upstream tank showing the small individual traps.

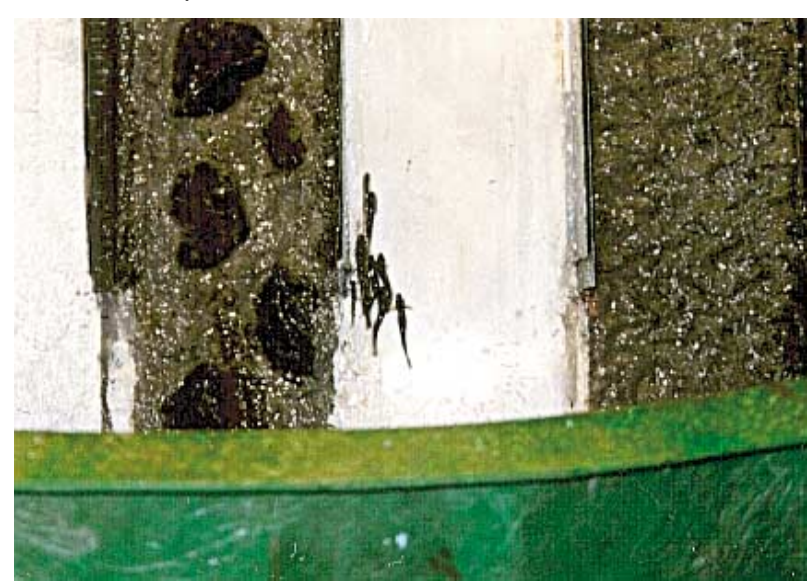

Photo 5 :vue d'un groupe de Sicyopterus lagocephalus remontant le substrat PVC.

Photo 5 : view of a group of Sicyopterus lagocephalus climbing the PVC substrate.

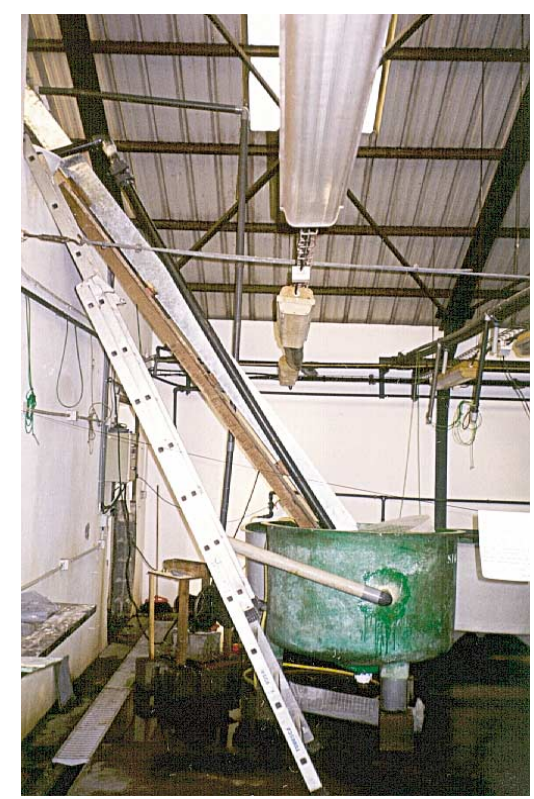

Photo 2 : vue de profil du pilote expérimental à la pente de $50^{\circ}$. Photo 2 : side view of the test installation at $50^{\circ}$ slope.

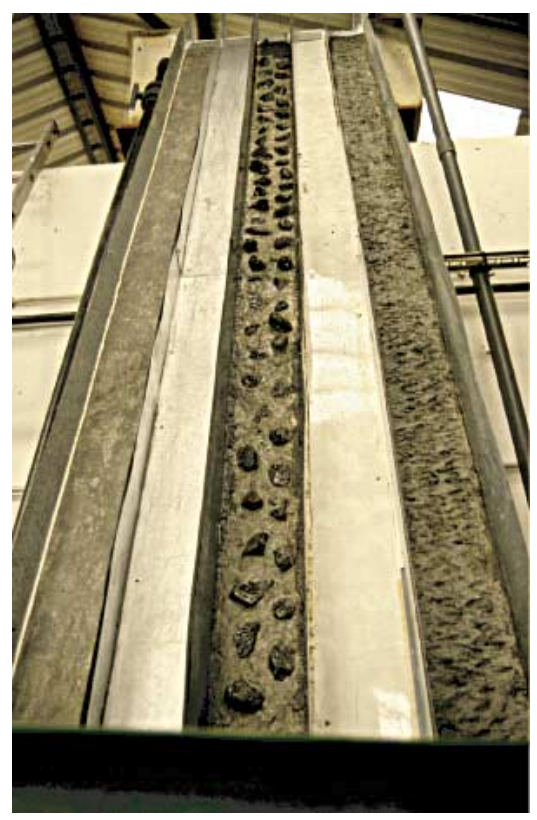

Photo 3 : vue de face du pilote expérimental à la pente de $50^{\circ}$. Photo 3 :front view of the test installation at $50^{\circ}$ slope. 